\title{
Influence of Bearing Structure Parameter Changes on Vehicle Modal Characteristics
}

\author{
Xiao-Long HE ${ }^{1, a}$, Li-Min ZHANG ${ }^{1 *}$, Lian-Tao LU ${ }^{1}$, Fei-Li QIU ${ }^{1}$, \\ Wei-Guang SUN², Ai-Qin TIAN² \\ ${ }^{1}$ Traction State Key Laboratory, Southwest Jiaotong University, Chengdu 610031, China \\ ${ }^{2}$ CSR Sifang Co.Ltd, Qingdao Shandong 266000, China \\ aHexiaolong_vip@163.com, *zhang-Im01@163.com \\ ${ }^{*}$ Corresponding author
}

Keywords: Material Thickness, Vehicle Body, Modal Frequencies, Vertical Bending Nodes, Ratio of Vertical Bending and Torsion.

\begin{abstract}
It is known that vehicle structural thickness change will lead to vehicle modal characteristics change .In this paper, the thickness of floor, sidewalls and vehicle top are chosen as the variables so as to calculate the relationship amongst thickness change and frequencies (Vertical bending- $f_{1}$, Torsion- $f_{2}$, Rhombus- $f_{3}$ ), vertical nodes and the ratio of Vertical bending and Torsion respectively. The results show that structure size have a significant impact on the vehicle body natural frequency; In terms of the overall degree of influence, the vehicle top and vehicle top corrugated board have greater influence on $f 1, f 2, f 3$ than the other size's; For a given vehicle, in the premise of no significant increase in vehicle body weight, we can increase the natural frequencies $f 1$, $f 2, f 3$ by adjusting the dimensions of the material. Also we can change the vehicle body vertical bending nodes by adjusting the component dimensions, and increase the thickness of the vehicle top to improve the ratio of vehicle body vertical bending and torsion, and reduce the ratio of vehicle body vertical bending and torsion by increase the floor, sidewall thickness. But how the floor thickness affect the ratio of vertical bending and torsion depends on thickness which has been designed.
\end{abstract}

\section{Introduction}

With the increasing train speed, the vertical, longitudinal, lateral and tensional excitation which is loaded on the train in operation is increasing [1]. On the other hands, because of the application of lightweight train design[2], following with reducing natural frequency, this leads to the train generates more vibration. This will not only reduce the ride comfort, but also it will affect the service life of the structure.

The thickness of the body structure change will lead to changes in the stiffness and mass of [3], the deformation stiffness of the control structure of the vehicle body (deflections), the stability and natural frequency. The thickness of the body structure change will lead to changes in the stiffness and mass [3]. And the stiffness of the body controls the structure deformation, stability and natural frequency of the vehicle body.

\section{The Vehicle Body Finite Element Model and the Choose of Design Variables}

\section{The Vehicle Body Finite Element Model}

The geometric model was divided into meshes by HYPERMESH [4].In this paper the vehicle model was dispersed with SHELL63 elements. The vehicle was divided into 661979 units and 501639 nodes. The real constant of each part of the vehicle body was defined according to the actual thickness parameters. The finite element model of vehicle body is shown in figure 3.1.

The vehicle bearing structure was mainly composed of floor, sidewalls, vehicle top and end walls [5].In this paper, the floor of certain high speed vehicle was mainly established by spandrel beam, 
traction beam, the corrugated floor and the air guide etc... The spandrel beam edge that is the longitudinal beam on the left and the right of the floor and is the key components of connecting the floor and side wall. The corrugated floor is made of the extrusion-shaped aluminum. Also the reinforced frame is added in the longitudinal direction in order to enhance the longitudinal strength of the floor (Figure 3.2).In the calculation we ignore the structure dimension part which has few effect, and only consider the effect of corrugated floor on modal characteristics of the vehicle body.

For the sidewalls, we only modify the thickness of inner sidewalls and corrugated board because of considering the limiting width through body design throughout the calculation. The model of the sidewalls is shown in figure 3.3. The vehicle top is composed of flat, round top and other structures, and the main components are butted, installed and welded using the same large-scale hollow extruded shape welded splice. In the analysis, we only consider the influence of the thickness of flat part and the corrugated board on the body modal characteristic. Because of the vehicle gauge and other factors, we only consider the effct of thickness of inner sidewalls. The model of vehicle top is shown in figure 3.4.
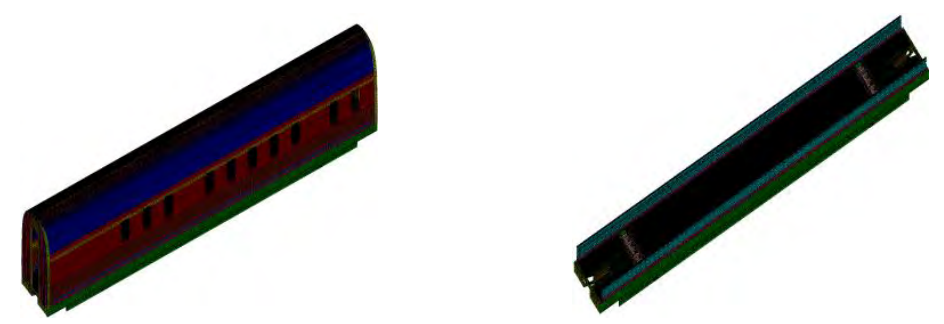

Fig.3.1 Vehicle Body Finite Element Model Fig.3.2Floor Model

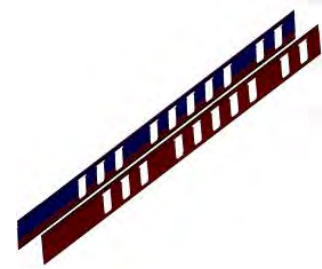

Fig.3.3 Sidewalls Model

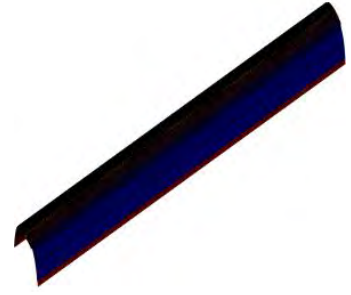

Fig.3.4Vehicle Top Model

\section{The Choose of Design Variables}

We make modal analysis and calculation for the vehicle using the Block Lanczos method in ANSYS [6], and extract the natural frequency and mode of vibration within the $30 \mathrm{~Hz}$.in order to guarantee the effectiveness of the model, we compare the calculate result and experimental results. The results are shown in table 3.1 .

Tab. 3.1 Calculation and Experimental Results

\begin{tabular}{ccccc}
\hline Orders & $\begin{array}{c}\text { Calculation } \\
\text { Frequencies }[\mathrm{Hz}]\end{array}$ & $\begin{array}{c}\text { Experimental } \\
\text { Frequencies }[\mathrm{Hz}]\end{array}$ & Modal shapes & Errors[\%] \\
\hline 1 & 21.631 & 21.2 & Vertical bending & 2.0 \\
\hline 2 & 22.967 & 22.8 & Rhombus & 0.7 \\
\hline 3 & 38.115 & 38.0 & Torsion & 0.3 \\
\hline
\end{tabular}

The original thickness of the floor is $2.5 \mathrm{~mm}$.In the original design model, the inner and outer side floor have the same thickness. So the range of the thickness of the selected floor d1 is from $1 \mathrm{~mm}$ to $5.5 \mathrm{~mm}$. The range of the thickness of inner side $\mathrm{d} 3$ is from $1.5 \mathrm{~mm}-6 \mathrm{~mm}$. The inner thickness range of the vehicle top $\mathrm{d} 5$ is from $1 \mathrm{~mm}$ to $5.5 \mathrm{~mm}$.All the corrugated boards have the same thickness $(1.8 \mathrm{~mm})$ and their range of thickness are all from $1 \mathrm{~mm}$ to $3 \mathrm{~mm}$. Calculation condition table as shown in Table 3.2. 
Tab. 3.2 Calculation Condition

\begin{tabular}{ccccccccccc}
\hline Material location & $d-1.5$ & $d-1$ & $d-0.5$ & $d$ & $d+0.5$ & $d+1$ & $d+1.5$ & $d+2$ & $d+2.5$ & $d+3$ \\
\hline Floor $\left(d_{1}\right)[\mathrm{mm}]$ & 1 & 1.5 & 2 & 2.5 & 3 & 3.5 & 4 & 4.5 & 5 & 5.5 \\
Sidewall $\left(d_{3}\right)[\mathrm{mm}]$ & 1.5 & 2 & 2.5 & 3 & 3.5 & 4 & 4.5 & 5 & 5.5 & 6 \\
Vehicle top $\left(d_{5}\right)[\mathrm{mm}]$ & 1 & 1.5 & 2 & 2.5 & 3 & 3.5 & 4 & 4.5 & 5 & 5.5 \\
\hline Corrugated sheet & $d-0.8$ & $d-0.6$ & $d-0.4$ & $d-0.2$ & $d$ & $d+0.2$ & $d+0.4$ & $d+0.6$ & $d+1$ & $d+1.2$ \\
\hline$\left(d_{2}, d_{4}, d_{6}\right)[\mathrm{mm}]$ & 1 & 1.2 & 1.4 & 1.6 & 1.8 & 2 & 2.2 & 2.4 & 2.8 & 3 \\
\hline
\end{tabular}

\section{The Calculate Effect Result of Structural Parameters Change on Modal Characteristics}

\section{The Effect of Bearing Structural Parameters on Modal Frequencies}

By finite element calculation, the influence of vehicle body structural parameters on vertical bending, torsion and rhombus frequencies outcome as shown in Fig.4.1, 4.1 and 4.3 respectively.

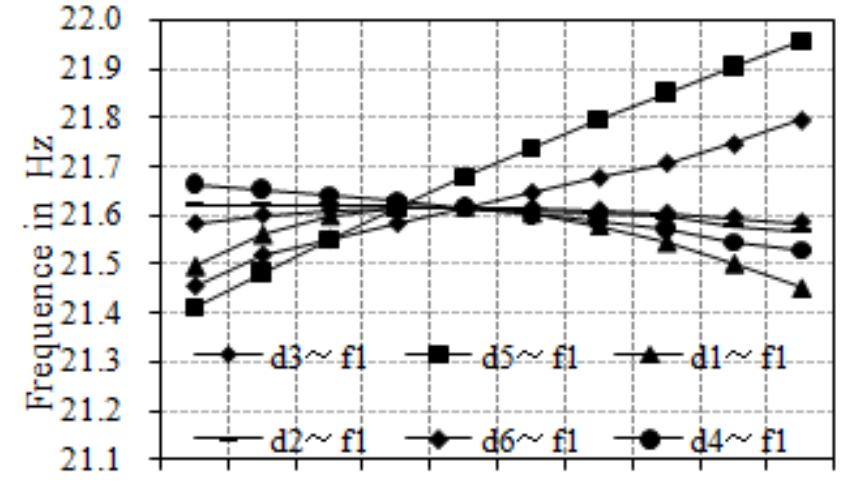

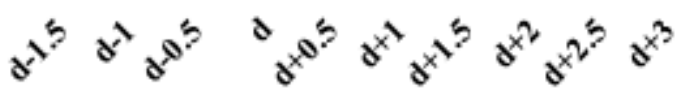

Thickness in $\mathrm{mm}$

Fig.4.1 The Relationship between Material Thickness and Vertical Bending Frequencies

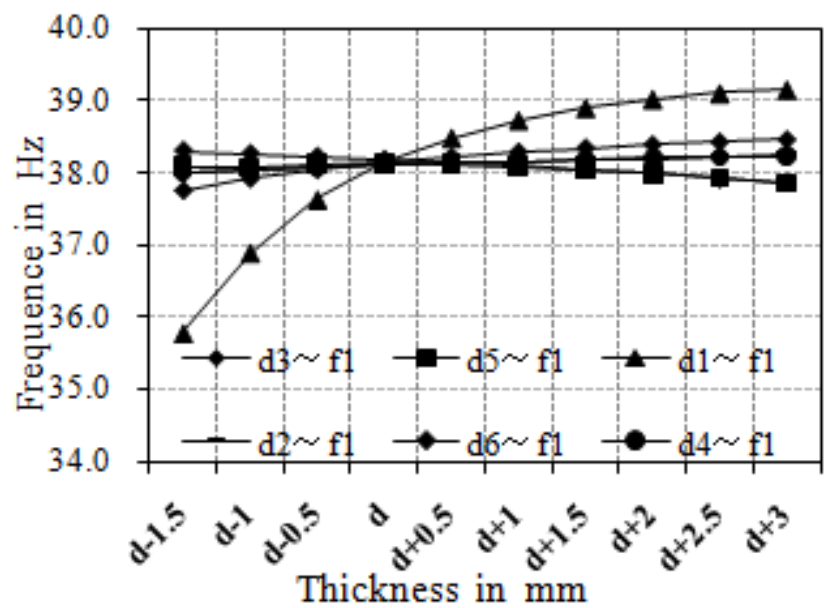

Fig.4.3The Relationship between Material Thickness and Torsion Frequencies
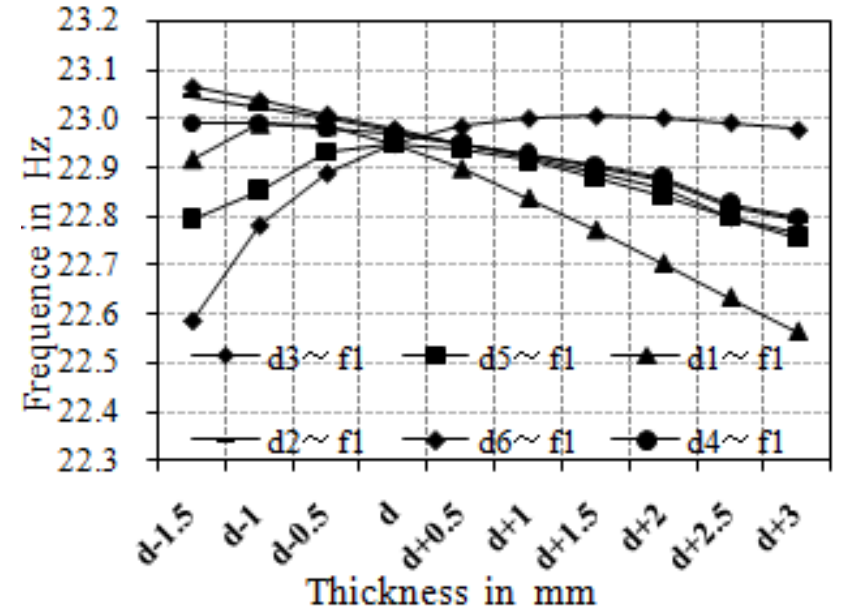

Fig.4.2 The Relationship between Material Thickness and Rhombus Frequencies

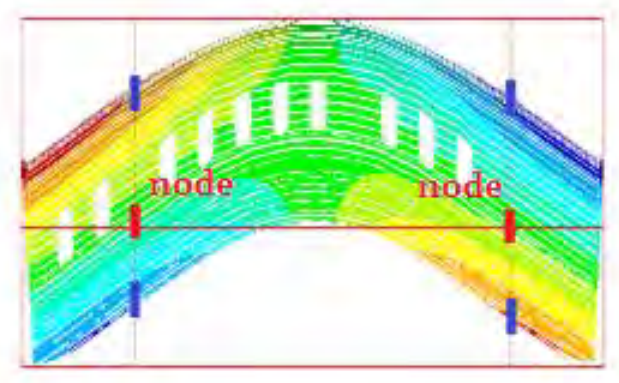

Thicknessin $\mathrm{m}$

Fig.4.4 The Vehicle Body Vertical Bending Vibration Figure

Figure 4.1 shows: The curve of top thickness $\sim$ fl has the largest gradient value. That is the vehicle top has the largest influence degree on $f_{l}$, followed by the top corrugated boards. It has little help to improve $f_{l}$ by increase the thickness of floors, the floor corrugated boards, the side walls and the sidewalls corrugated boards. But reducing the thickness of floor, sidewalls, vehicle top and top 
corrugated boards will reduce $f_{1}$.And reducing the sidewalls and sidewalls corrugated boards will increase $f_{l}$.

Figure 4.2shows: Increasing the thickness of all boards will reduce the frequency of rhombus. The curve of sidewalls $\sim f_{2}$ has the smallest gradient value, the curve of floor $\sim f_{2}$ has the largest gradient value. Other material thickness has similar influence degree on the rhombus frequency. However, reducing the thickness of corrugated boards will increase the rhombus frequency, and reducing thickness of remaining material will reduce rhombus frequency.

Figure 4.3 shows:the curve of floor $\sim f_{3}$ has the largest gradient value. That is the floor thickness has the largest influence degree on $f_{3}$.the remaining material has little influence on $f_{3}$.

\section{The Influence of Bearing Structural Design Variables on Body Modal Shape}

The fixed points on the vehicle body are named nodes when vehicle body vertical bending deformation of occurs. The position of nodes has particular importance on the hanging devices chosen and installation. The vertical bending mode shape is shown in Figure 3.4.

The research methods are as follows: First, selecting the nodes which has zero lateral and vertical displacement from the neutral plane in the calculation result and noting the node number and longitudinal position coordinates. Then select and node the zero-displacement nodes from other calculation condition results.

Finally, horizontal line is chosen to represents the undeformed equilibrium position, And choose the end and intermediate position nodes as the feature points to obtain schematic diagram of how the material thickness influence the vertical bending nodes.

The calculation result as shown in table4.1. Where $l_{1}, l_{2}$ denote the distance between each node and the vehicle body end.

Tab. 4.1Caculation Results

\begin{tabular}{|c|c|c|c|c|c|}
\hline \multicolumn{2}{|c|}{ Condition } & $x$ coordinate of left node & $l_{1}$ & $r$ coordinate of right node & \multirow{2}{*}{$\frac{l_{2}}{5.334}$} \\
\hline \multirow{3}{*}{$\mathrm{d}-1.5[\mathrm{~m}]$} & Floor & 5.128 & 5.128 & 18.766 & \\
\hline & Sidewalls & 5.228 & 5.228 & 18.849 & 5.251 \\
\hline & Vehicle top & 5.198 & 5.198 & 18.798 & 5.302 \\
\hline \multirow{3}{*}{$\mathrm{d}-1[\mathrm{~m}]$} & Floor & 5.152 & 5.152 & 18.799 & 5.301 \\
\hline & Sidewalls & 5.224 & 5.224 & 18.850 & 5.25 \\
\hline & Vehicle top & 5.202 & 5.202 & 18.807 & 5.293 \\
\hline \multirow{3}{*}{$\mathrm{d}+1[\mathrm{~m}]$} & Floor & 5.224 & 5.224 & 18.872 & 5.228 \\
\hline & Sidewalls & 5.218 & 5.218 & 18.842 & 5.258 \\
\hline & Vehicle top & 5.219 & 5.219 & 18.819 & 5.281 \\
\hline \multirow{3}{*}{$\mathrm{d}+2[\mathrm{~m}]$} & Floor & 5.257 & 5.257 & 18.892 & 5.208 \\
\hline & Sidewalls & 5.209 & 5.209 & 18.843 & 5.257 \\
\hline & Vehicle top & 5.223 & 5.223 & 18.825 & 5.275 \\
\hline \multirow[t]{3}{*}{$\begin{array}{l}5.35 \\
5.30\end{array}$} & & $\square \mathrm{d}-1.5=0$ & $-1 \square d$ & $+1 \square d+2$ & \\
\hline & $\mathbb{B}$ & $B$ & 8 & B & \\
\hline & $t_{1-}$ & $\begin{array}{l}l_{1-\text { Sidewalls }} \\
\text { Material lo }\end{array}$ & $\begin{array}{l}l_{2-S i d e w} \\
\text { antón }\end{array}$ & 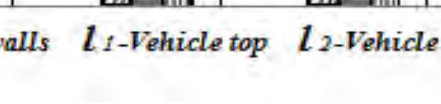 & \\
\hline
\end{tabular}

Fig.4.4 The Trend Figure of Distance between Body End and Node 

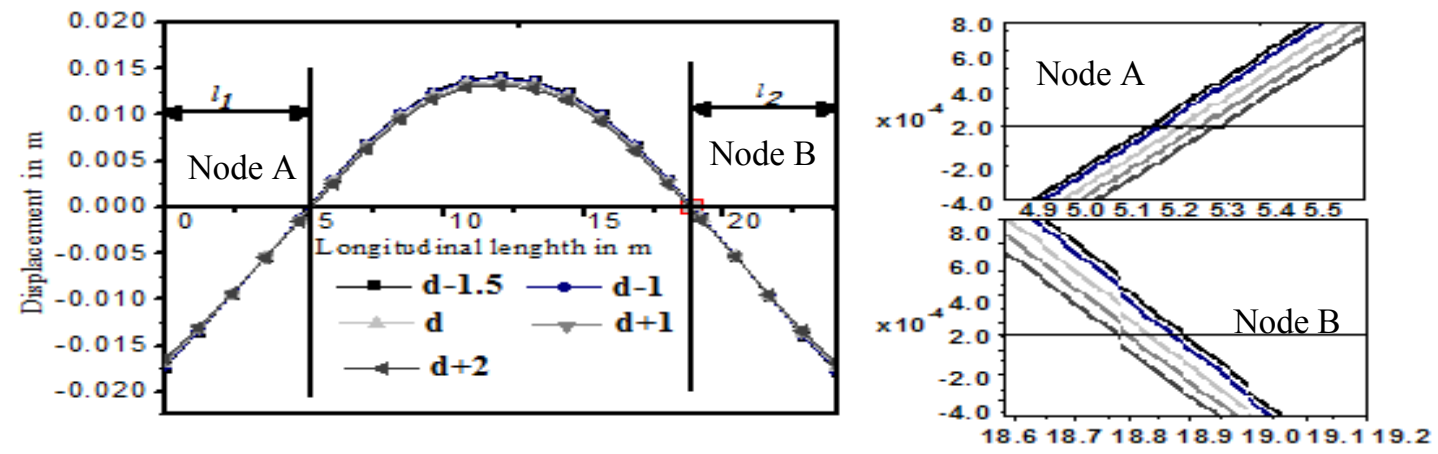

Fig. 4.5 The Effect of the Thickness of Floor on Vertical Bending Node Location of Vehicle Body and Its Nodes Thinning Curve

Figure 4.5 shows: with the increase of the inside floor thickness, vehicle body vertical bending nodes gradually move to the middle of car, and the right nodes move obvious to the lefts; Figure 3.6 shows: with the increase of the flank thickness, the left side nodes move to the left side of car, the location of right side node basically unchanged; Figure 3.7 shows: with the increase of the thickness of the roof, vehicle body vertical bending nodes gradually move to the middle of car, and the right nodes move obvious to the lefts.

\section{The Influence of the Thickness of the Bearing Structure to the Ratio of Vehicle Body Bending and Twisting Frequency}

The ratio of vehicle body bending and twisting frequency [7][8] is an important factor to take into account for car design, a rational design can effectively avoid coupled vibration caused by the two frequencies close too much, the lower the two frequencies, the smaller energy consumption for self-excited vibration, and more easily flutter; if the bending, twisting frequency closer, not only the energy exchange turn more easily, but also easy to flutter. According to the results of the previous section, the empirical formulas about the relationship between the thickness of each bearing structure and the bending, diamond, torsion natural frequency and modal frequencies of the vehicle body by polynomial regression method are as follows:

$$
\begin{aligned}
& A_{1}=\frac{f_{1}}{f_{3}}=\frac{21.2965+0.0034 d_{1}-0.0602 d_{1}{ }^{2}+0.25736 d_{1}{ }^{3}}{32.8345+0.0549 d_{1}-0.763 d_{1}{ }^{2}+3.6913 d_{1}{ }^{3}} . \\
& A_{3}=\frac{f_{1}}{f_{3}}=\frac{21.4871+0.0011 d_{3}-0.0185 d_{3}^{2}+0.0889 d_{3}^{3}}{37.0847+0.0048 d_{3}-0.0837 d_{3}^{2}+0.5591 d_{3}^{3}} \\
& A_{5}=\frac{f_{1}}{f_{3}}=\frac{21.2602-0.0072 d_{5}^{2}+0.1587 d_{5}^{3}}{37.9511-0.0363 d_{5}^{2}+0.1553 d_{5}^{3}} .
\end{aligned}
$$

The calculation results are shown in figure 5.1 to 5.3

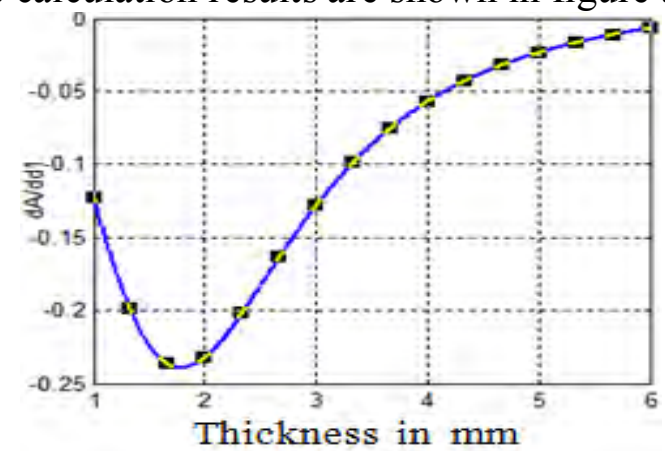

Fig. 5.1 The Bending and Twisting Frequency Ratio Influenced by the Thickness of Floor

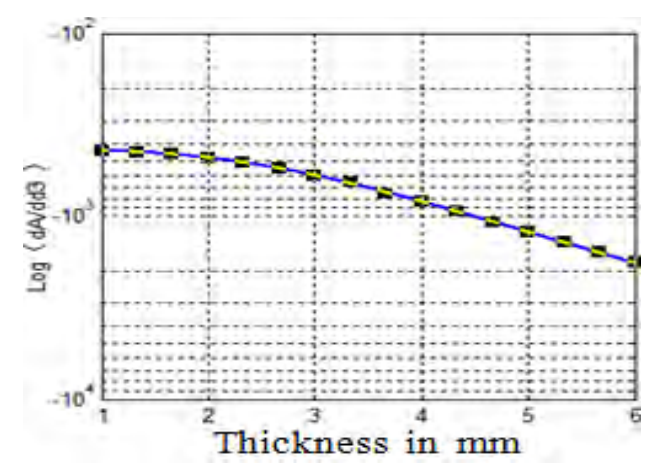

Fig. 5.2 The Bending and Twisting Frequency Ratio Influenced by the Thickness of Sidewalls 


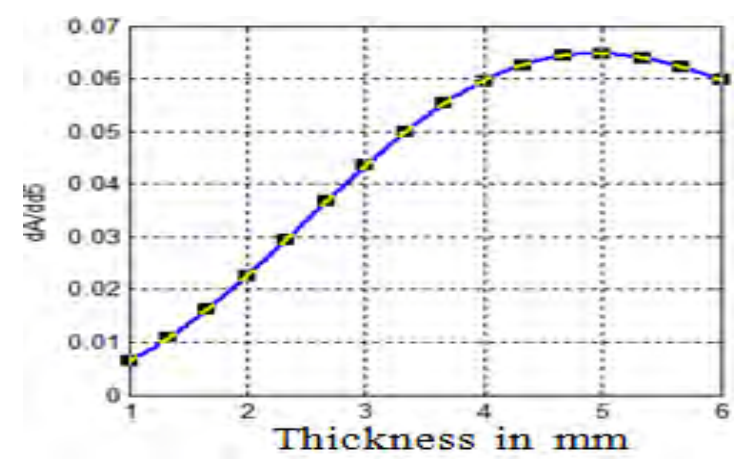

Fig. 5.3 The Bending and Twisting Frequency Ratio Influenced by the Thickness of Top

Figure5.1 shows: the bending and twisting frequency ratio turn smaller with increasing of thickness; when the floor thickness less than $3 \mathrm{~mm}$, the ratio change rapidly with thickness. The ratio reach maximum when the thickness is $1 \mathrm{~mm}$.Figure 5.2 shows: with the increase in the thickness of the body sidewall, the bending and twisting frequency ratio decreases monotonically, and the reduced amplitude turn smaller.Figure 5.3 shows: the bending and twisting frequency ratio turn bigger with increasing the roof thickness; after the roof thickness greater than $4 \mathrm{~mm}$, the ratio to the roof thickness shows an approximate linear increasing trend.

\section{Summary}

Profile structure size has significant effect on the natural frequencies $f_{1}, f_{2}, f_{3}$ of vehicle body, In terms of the overall impact, the dimension of roof, roof of corrugated board and floor is greater than the influence of other sizes to $f_{1}, f 2, f 3$; for a certain vehicle body, under the premise without a significant increase the weight, it can adjust the size of the parts to improve the body's natural frequency $f_{1}, f_{2}, f_{3}$, and change the location of bending node; Increasing the roof thickness can improve the ratio of bending and twisting frequency, increase the floor, side wall thickness can reduce the ratio. But the influence of floor to the bending and twisting frequency ratio is decided on the thickness of it.

\section{References}

[1]Luo Ren, Zeng Jing, Modeling and Ride Quality Analysis of Railway Train System, J. China railway science, Vol.127, No.11, (2006)72-77.

[2]Huang Wen yang, Indistinct Features of Railway High Speed Train in Early Years of the 21th Century, railway locomotive \&car, J. Vol.29No.2(2009)23-30.

$[3,7] \mathrm{Hu}$ Yong, Fan Huabing, on calculation of bearing capacity of concrete member subjected to bending torsion with consideration of related influence, J. Engineering design and construction, Vol.37No.5 (2005)8-12.

[4]Xiong Zhenbing, Luo Huixin, Preprocessing technology of FEA based on Hyper Mesh software, J. Drainage and Irrigation Machinery, Vol. 24 No. 3(2006)35-38.

[5]Zhao Honglun, Yu Chengliang, Study on Optimization Design of Car body Structure, J. High speed Maglev Train, Vol. 29, No. 4(2007)43-47.

[6]Hua Dai, Preconditioning block lanczos algorithm for solving symmetric Eigen value problems, J. Journal of Computational Mathematics, Vol.18, No.4, (200 0)365-374.

[8]XU Yu-ye, HE Ye, Wang Quanfeng, Study on the torsion performances of concrete specially shaped columns under the actions of compression, bending, shear and torsion, J. engineering mechanics, Vol.31 No.6, (2014)101-109. 